\title{
EMPLEO DE PLANTAS MEDICINALES EN USUARIOS DE DOS HOSPITALES REFERENCIALES DEL CUSCO, PERÚ
}

\author{
Gladys Oblitas ${ }^{1, a}$, Gustavo Hernández-Córdova ${ }^{1, b}$, Analí Chiclla ${ }^{1, c}$, María Antich-Barrientos $^{1, b}$, \\ Lucero Ccorihuamán-Cusitito ${ }^{1, b}$, Franco Romaní2,3d
}

\section{RESUMEN}

Con el fin de determinar la frecuencia de empleo de plantas medicinales y describir las características de su uso en pacientes de dos hospitales referenciales de la ciudad de Cusco, se realizó un estudio transversal entre agosto y septiembre de 2011. Para el recojo de datos se construyó un instrumento, validado por juicio de expertos. El tamaño de la muestra fue de 250 personas seleccionadas no probabilísticamente. El 83,2 y 75,3\% informaron haber empleado plantas medicinales alguna vez en su vida y en el último mes, respectivamente; además, el 85,7 señaló que desearía que su médico le recete plantas medicinales. Sus usos más frecuentes son para problemas digestivos (62,4\%); urinarios $(42,4 \%)$, y respiratorios $(40,4 \%)$. Se concluye que el empleo de plantas medicinales se encuentra bastante difundido entre los usuarios de dos hospitales referenciales de la ciudad de Cusco. Los patrones de empleo revelan que los pacientes desean que los médicos del sistema de salud prescriban plantas medicinales en su acto médico.

Palabras clave: Plantas medicinales; Medicina tradicional; Hospitales; Perú (fuente: DeCS BIREME).

\section{USE OF MEDICINAL PLANTS AMONG PEOPLE ATTENDING TWO REFERENCE HOSPITALS IN CUSCO, PERU}

\section{ABSTRACT}

In order to determine the frequency and characteristics of the use of medicinal plants in patients from two third-level hospitals in the city of Cusco, a cross-sectional study was conducted between August and September 2011. For data collection, an instrument was built and validated through experts' judgment. The sample included 250 people selected in a non-probabilistic way. 83.2 and $75.3 \%$ informed having had used medicinal plants sometime during their lives and in the last month, respectively; additionally, 85.7 indicated that they wished their doctor would have prescribed them medicinal plants. Their most frequent uses include digestive problems $(62.4 \%)$ as well as urinary $(42.4 \%)$ and respiratory problems $(40.4 \%)$. We conclude that the use of medicinal plants is widely spread among users of two hospitals in the city of Cusco. Utilization patterns show that patients wished the physicians of the health system prescribed medicinal plants in their consults.

Key words: Plants, medicinal; Medicine, traditional; Hospitals; Peru (source: MeSH NLM).

\section{INTRODUCCIÓN}

Según la Organización Mundial de Salud, la medicina tradicional comprende a las prácticas, los enfoques, los conocimientos y las creencias sanitarias diversas que incorporan medicinas basadas en plantas; en animales o minerales; en terapias espirituales; en técnicas manuales, y en ejercicios aplicados de forma individual, o en combinación, para mantener el bienestar, además de tratar, diagnosticar y prevenir las enfermedades ${ }^{(1)}$.

En países en vías de desarrollo se ha descrito que hasta el $90 \%$ de la población usa la medicina tradicional ${ }^{(1)}$.
Estudios en Latinoamérica reportan diferentes frecuencias de uso de plantas medicinales. En una zona rural de Córdova (Argentina) se ha descrito que el $100 \%$ de la población conocía sobre el uso de plantas medicinales, y el $78 \%$ conocía al menos cinco especies (2). En Brasil, dependiendo de la región, la frecuencia de uso de plantas medicinales puede variar entre 70 y $98 \%$ de pobladores que la usan ${ }^{(3,4)}$. En tanto que en Panamá el $84 \%$ de adultos que acuden a una unidad de atención primaria, usan también plantas medicinales ${ }^{(5)}$.

El año 2007 se realizó una cumbre internacional organizada por el Colegio Médico del Perú, conocida

\footnotetext{
Universidad Nacional de San Antonio Abad de Cusco. Cusco, Perú.

Oficina General de Investigación y Transferencia Tecnológica, Instituto Nacional de Salud. Lima, Perú.

Departamento Académico de Medicina Preventiva y Salud Pública, Universidad Nacional Mayor de San Marcos. Lima, Perú.

a Médico salubrista, ${ }^{\mathrm{b}}$ estudiante de Medicina, ${ }^{\mathrm{c}}$ estudiante de Biología, ${ }^{\mathrm{d}}$ médico cirujano

Recibido: 29-11-12 Aprobado: 06-02-13
}

Citar como: Oblitas G, Hernández-Córdova G, Chiclla A, Antich-Barrientos M, Ccorihuamán-Cusitito L, Romaní F. Empleo de plantas medicinales en usuarios de dos hospitales referenciales del Cusco, Perú. Rev Peru Med Exp Salud Publica. 2013;30(1):64-8. 
como La Declaración de Lima, la cual reconoce -entre otros puntos- la importancia de la medicina tradicional y recomienda su armonización y articulación con los sistemas de salud oficiales de cada país ${ }^{(6)}$. La medicina occidental y tradicional no son excluyentes sino que coexisten dependiendo de la disponibilidad de los servicios de salud. (7) En el Perú, el tema ha sido trabajado mediante varios estudios de etnobotánica realizados en poblaciones diversas ${ }^{(7-10)}$. Sin embargo, no encontramos estudios sobre la frecuencia de empleo de plantas medicinales ni en la población en general ni entre aquellos que acuden a hospitales del sistema de salud oficial, especialmente al tercer nivel de atención, donde existe una mayor oferta de servicios de salud (que incluye medicamentos, terapias y servicios disponibles) que en zonas netamente rurales.

Las explicaciones para el uso extendido de plantas medicinales en el Perú obedecen a la riqueza y variedad en especies vegetales ${ }^{(11)}$, y a la tradición existente sobre su empleo desde el periodo preinca, y que ha persistido hasta la fecha ${ }^{(9,12)}$. En nuestro país el uso de plantas medicinales coexiste con la medicina occidental, especialmente en el primer nivel de atención, donde su uso está orientado a prevenir y aliviar diversos problemas de salud ${ }^{(7)}$; sin embargo, se ha observado que su uso persiste aún entre los usuarios de establecimientos de salud de mayor capacidad resolutiva ${ }^{(13)}$.

La Dirección Regional de Salud del Cusco está compuesta por cuatro redes de servicios de salud y dos hospitales referenciales; la ciudad del Cusco se caracteriza por su riqueza cultural, por sus tradiciones y su folclore, entre los que se cuenta la medicina tradicional; a pesar de ello, no hemos encontrado publicaciones que sugieran las características del consumo, o las motivaciones para el empleo de plantas medicinales; particularmente en pobladores de zonas andinas. Por ello, el objetivo del presente estudio fue determinar la frecuencia $y$ las características del empleo de plantas medicinales en población adulta que asiste a los dos hospitales referenciales de una ciudad capital de la sierra peruana.

\section{EL ESTUDIO}

Se realizó un estudio transversal en la ciudad de Cusco entre los meses de agosto y septiembre de 2011, se incluyeron dos hospitales de referencia de la zona (el Hospital de Apoyo Departamental nivel III del Ministerio de Salud [HADIII] y el Hospital Nacional Adolfo Guevara Velasco nivel IV del Seguro Social [HNAGV]).

La población estuvo conformada por los pacientes que acuden a consulta externa de medicina general y medicina interna de los dos hospitales. El tamaño planeado de la muestra fue de 250 personas, para su cálculo se consideró un tamaño poblacional no conocido, una prevalencia de uso de plantas medicinales del $84 \%$ (6), el nivel de confianza se fijó en $95 \%$, el error en $5 \%$ y la tasa anticipada de respuesta en $80 \%$. Los participantes fueron elegidos de manera no probabilística, por cuotas (125 por hospital), los cuales fueron seleccionaron a través de la ocupación de asientos preescogidos en la sala de espera. Se incluyeron usuarios de los hospitales mayores de edad y que aceptaran participar del estudio luego de haber obtenido el consentimiento informado oral. Se excluyeron los cuestionarios incompletos en más del $40 \%$ de las preguntas o que no respondieron a la pregunta sobre uso de plantas medicinales.

Para la recolección de datos se construyó un cuestionario estructurado, el cual incluyó características sociodemográficas, nivel socioeconómico y preguntas relacionadas al uso de plantas medicinales. El nivel socioeconómico fue determinado mediante la escala de Graffar-Mendez, la cual lo clasifica en cinco estratos (alto, medio alto, medio, pobreza relativa y pobreza crítica). La validez de contenido fue evaluado mediante juicio de expertos, en el que participaron expertos en medicina tradicional, Salud Pública, medicina familiar y medicina interna, la viabilidad del instrumento fue evaluada con una prueba piloto. El cuestionario fue traducido al idioma quechua y fue aplicado por un personal bilingüe de ciencias de la salud (españolquechua). Se realizó la indicación a los participantes de no considerar como uso de planta medicinal a productos sintéticos y transformados como bebidas en sobres filtrantes, extractos comerciales o multivitamínicos.

El estudio fue aprobado en la Facultad de Medicina de la Universidad Nacional San Antonio Abad de Cusco. Se empleó un consentimiento informado oral previa participación. El análisis estadístico incluyó el cálculo de las frecuencias absolutas y relativas de las variables en estudio, para ello se empleó el programa estadístico SPSS Ver 19.0.

\section{HALLAZGOS}

Se encuestaron un total de 250 personas, la media de edad fue de $42,4 \pm 15,9$ años, el $66 \%$ fueron mujeres. El $46,8 \%$ tenía educación universitaria y el $70,1 \%$ de ellos fue captado en el HNAGV; el 10\% tenía educación primaria o menor y el $88 \%$ de ellos fue captado en el HADIII. El $22 \%$ pertenecía a la clase media alta, de ellos, el 83,6\% correspondía al HNAGV; mientras que el $12 \%$ se encontraba en pobreza crítica, de los cuales el $86,7 \%$ fue captado en el HADIII (Tabla 1). Todos los participantes respondieron el cuestionario en español. 
Tabla 1. Características sociodemográficas de los participantes según hospital.

\begin{tabular}{|c|c|c|}
\hline & \multicolumn{2}{|c|}{ Hospital } \\
\hline & $\begin{array}{l}\text { MINSA } \\
\text { N. }{ }^{\circ}(\%) \\
\end{array}$ & $\begin{array}{c}\text { EsSalud } \\
\mathrm{N} .^{\circ}(\%)\end{array}$ \\
\hline \multicolumn{3}{|l|}{ Sexo* } \\
\hline Masculino & $43(51,2)$ & $41(48,8)$ \\
\hline Femenino & $82(49,7)$ & $83(50,3)$ \\
\hline \multicolumn{3}{|l|}{ Nivel educativo } \\
\hline Primaria o menos & $22(88,0)$ & $3(12,0)$ \\
\hline Secundaria & $38(71,7)$ & $15(28,3)$ \\
\hline Técnica Superior & $30(54,5)$ & $25(45,5)$ \\
\hline Universitaria & $35(29,9)$ & $82(70,1)$ \\
\hline \multicolumn{3}{|c|}{ Estrato Socioeconómico ${ }^{\dagger}$} \\
\hline Media alta & $9(16,4)$ & $46(83,6)$ \\
\hline Media & $20(28,6)$ & $50(71,4)$ \\
\hline Pobreza relativa & $50(68,5)$ & $23(31,5)$ \\
\hline Pobreza crítica & $26(86,7)$ & $4(13,3)$ \\
\hline
\end{tabular}

*Un caso de EsSALUD no registró su sexo. ${ }^{\dagger}$ veinte casos del MINSA

y dos casos de EsSALUD no completaron la escala de Graffar-Mendez

El $83,2 \%$ reportó haber usado plantas medicinales, el promedio de la edad entre los que emplean plantas medicinales fue de 42,4 $\pm 16,1$ años. Las plantas medicinales fueron usadas con mayor frecuencia para tratar problemas digestivos $(62,4 \%)$, urinarios $(42,2 \%)$ y respiratorios $(40,4 \%)$. Los principales motivos por el cual usaron alguna vez plantas medicinales fueron por el consejo de familiar o amigo $(47,6 \%)$ y por su confianza en la medicina tradicional $(29,6 \%)$. El $36,8 \%$ de participantes afirmó que su médico le recetó plantas medicinales en alguna oportunidad, el $67,8 \%$ indicó que practica el uso combinado de medicamentos y plantas medicinales, además al 93,7\% le gustaría que su médico se las recete. Es importante destacar que el $75,3 \%$ cree que las plantas medicinales pueden producir daño si no son empleadas correctamente; mientras que $60,9 \%$ tiene la percepción que las plantas medicinales si son usadas adecuadamente causan menor daño en la salud que los medicamentos empleados en la medicina occidental (Tabla 2).

La proporción de empleo de plantas medicinales entre aquellos que desean que el médico se las recete $(85,7 \%)$ es mayor que en el grupo que no lo desean $(60,0 \%)$. La proporción de uso de plantas medicinales entre aquellos cuyo médico les recomendó su empleo $(89,9 \%)$ es mayor que en el grupo donde no hubo esta recomendación $(79,7 \%)$. Entre los participantes que confían en la medicina tradicional la proporción del empleo de plantas medicinales fue mayor que en el grupo que no confían (98,6 y $76,6 \%$ respectivamente) (Tabla 3 ).

Un total de 215 participantes respondieron a la pregunta del número de veces que usaron plantas medicinales en el último mes. El 75,3\% refirió haberlas usado; el $43,7 \%$ de
Tabla 2. Patrón de empleo y percepción sobre las plantas medicinales.

\begin{tabular}{|c|c|}
\hline & N. ${ }^{\circ}(\%)$ \\
\hline \multicolumn{2}{|l|}{ Tipo de problema $(n=250)$} \\
\hline Problemas digestivos & $156(62,4)$ \\
\hline Problemas urinarios & $106(42,4)$ \\
\hline Problemas respiratorios & $101(40,4)$ \\
\hline Nervios & $38(15,2)$ \\
\hline $\begin{array}{l}\text { Problemas osteoarticulares y dolores de } \\
\text { cabeza }\end{array}$ & $33(13,2)$ \\
\hline Limpieza de sangre & $29(11,6)$ \\
\hline Problemas ginecológicos & $24(9,6)$ \\
\hline Problemas de piel y cabello & $19(7,6)$ \\
\hline \multicolumn{2}{|l|}{$\begin{array}{l}\text { Número de problemas en los que se usó plantas } \\
\text { medicinales }(n=208)^{*}\end{array}$} \\
\hline Uno & $61(29,3)$ \\
\hline Dos & $60(28.8)$ \\
\hline Tres & $53(25,5)$ \\
\hline Cuatro & $19(9,1)$ \\
\hline Cinco & $15(7,2)$ \\
\hline \multicolumn{2}{|l|}{ Motivo de uso de planta medicinal $(n=250)$} \\
\hline Consejo de familiar o amigo & $119(47,6)$ \\
\hline Confianza en medicina tradicional & $74(29,6)$ \\
\hline Para prevenir enfermedades & $48(19,2)$ \\
\hline Por publicidad/lectura revista/TV/Internet & $35(14,0)$ \\
\hline Complemento a las medicamentos /drogas & $34(13,6)$ \\
\hline Tratamiento de enfermedades no graves & $32(12,8)$ \\
\hline Consejo de su médico & $20(8,0)$ \\
\hline Consejo de herbolario, vendedor de hierbas & $12(4,8)$ \\
\hline Desconfianza en la medicina del hospital & $12(4,8)$ \\
\hline No acceso a otro tratamiento & $4(1,6)$ \\
\hline Consejo de farmacéutico & $4(1,6)$ \\
\hline \multicolumn{2}{|l|}{ Prácticas del proveedor de atención en salud } \\
\hline $\begin{array}{l}\text { Su médico le ha recetado plantas } \\
\text { medicinales }(n=242)\end{array}$ & $89(36,8)$ \\
\hline $\begin{array}{l}\text { Alguna vez un herbolario, curandero o } \\
\text { vendedor de plantas le ha recomendado } \\
\text { acudir al médico o farmacéutico }(n=238)\end{array}$ & $63(26,5)$ \\
\hline \multicolumn{2}{|l|}{ Prácticas del usuario } \\
\hline $\begin{array}{l}\text { Le gustaría que su médico le recete una } \\
\text { planta medicinal }(n=239)\end{array}$ & $224(93,7)$ \\
\hline $\begin{array}{l}\text { Cuando está enfermo toma una planta } \\
\text { medicinal además del medicamento } \\
\text { recetado por un médico }(n=239)\end{array}$ & $162(67,8)$ \\
\hline $\begin{array}{l}\text { Cree que algunas plantas medicinales } \\
\text { pueden producir daño si no son empleados } \\
\text { correctamente }(n=243)\end{array}$ & $183(75,3)$ \\
\hline \multicolumn{2}{|l|}{ Percepción sobre menor daño $(n=238)$} \\
\hline Tratamiento con plantas medicinales & $145(60,9)$ \\
\hline Tratamiento con medicamentos/drogas & $78(32,8)$ \\
\hline Ambos son igual de peligrosos & $15(6,3)$ \\
\hline
\end{tabular}

* El "n" en este caso corresponde a aquellos que afirmaron emplear plantas medicinales.

ellos las uso dos a menos veces; el 23,7\% las emplearon de tres a cinco veces; el $17,7 \%$ de 6 a 25 veces, y el $14,9 \%$ más de 25 veces. La mediana del número de veces de uso de plantas medicinales fue de 5 (rango intercuartil: 2-15). 
Tabla 3. Frecuencias de uso de plantas medicinales.

\begin{tabular}{|c|c|c|}
\hline & $\begin{array}{c}\text { Total } \\
\text { N. }\end{array}$ & $\begin{array}{c}\text { Usa } \\
\text { N. }{ }^{\circ}(\%)\end{array}$ \\
\hline \multicolumn{3}{|l|}{ Hospital (n=250) } \\
\hline EsSalud & 125 & $100(80,0)$ \\
\hline Ministerio de Salud & 125 & $108(86,4)$ \\
\hline \multicolumn{3}{|l|}{ Sexo $(n=249)$} \\
\hline Masculino & 84 & $66(78,6)$ \\
\hline Femenino & 165 & $141(85,5)$ \\
\hline \multicolumn{3}{|c|}{ Estrato socioeconómico $(\mathrm{n}=228)$} \\
\hline Media alta & 55 & $43(78,2)$ \\
\hline Media & 70 & $53(75,7)$ \\
\hline Pobreza relativa & 73 & $64(87,7)$ \\
\hline Pobreza crítica & 30 & $28(93,3)$ \\
\hline \multicolumn{3}{|l|}{ Grado de instrucción $(n=250)$} \\
\hline Primaria o menos & 25 & $22(88,0)$ \\
\hline Secundaria & 53 & $48(90,6)$ \\
\hline Técnico superior & 55 & $48(87,3)$ \\
\hline Universitario & 117 & $90(76,9)$ \\
\hline \multicolumn{3}{|c|}{$\begin{array}{l}\text { Recomendación de uso por médico } \\
(n=242)\end{array}$} \\
\hline Tiene recomendación & 89 & $80(89,9)$ \\
\hline No tiene recomendación & 153 & $122(79,7)$ \\
\hline \multicolumn{3}{|c|}{$\begin{array}{l}\text { Recomendación de uso por amigol } \\
\text { familiar }(n=250)\end{array}$} \\
\hline Tiene recomendación & 119 & $118(99,2)$ \\
\hline No tiene recomendación & 131 & $90(68,7)$ \\
\hline \multicolumn{3}{|c|}{ Deseo que el médico le recete $(n=239)$} \\
\hline Tiene el deseo & 224 & $192(85,7)$ \\
\hline No tiene el deseo & 15 & $9(60,0)$ \\
\hline \multicolumn{3}{|c|}{$\begin{array}{l}\text { Creencia que planta medicinal podría } \\
\text { causar daño }(n=243)\end{array}$} \\
\hline Cree & 183 & $152(83,1)$ \\
\hline No cree & 60 & $51(85,0)$ \\
\hline \multicolumn{3}{|c|}{$\begin{array}{l}\text { Confianza en medicina tradicional } \\
(n=250)\end{array}$} \\
\hline Confía & 74 & $73(98,6)$ \\
\hline No confía & 176 & $135(76,7)$ \\
\hline \multicolumn{3}{|c|}{$\begin{array}{l}\text { Confianza en medicamentos del } \\
\text { hospital }(n=250)\end{array}$} \\
\hline Confía & 12 & $12(100,0)$ \\
\hline No confía & 238 & $196(82,4)$ \\
\hline
\end{tabular}

Los valores de " $n$ " varían debido a que no se excluyeron del análisis los datos perdidos.

\section{DISCUSIÓN}

En el presente estudio se ha encontrado una elevada frecuencia de empleo de plantas medicinales en usuarios del tercer nivel de atención, dichas cifras son mayores a las prevalencias de uso en el último año reportada en países en vías de desarrollo como Jamaica $(72,6 \%)^{(14)}$ y Bolivia $(60 \%)^{(15)}$. Estos resultados confirman el amplio uso de plantas medicinales no solo en el primer nivel de atención, sino también en población que acude a establecimientos de mayor complejidad en diversas regiones o países ${ }^{(6,14,15)}$.

Algunos estudios sugieren que el uso de plantas medicinales está relacionado al sexo, a la edad, al nivel educativo, al nivel socioeconómico, a la religión y al tener pobre percepción de salud como características asociadas al uso de plantas medicinales ${ }^{(14-16)}$. En este estudio, al comparar el empleo entre ambos hospitales, a pesar que el nivel educativo y el nivel socioeconómico son mejores en el grupo del Hospital de EsSalud, las proporciones de uso solo difieren en 6,4\%. Esto sugeriría que el empleo de las plantas medicinales se encuentra altamente difundido en los usuarios de los servicios de salud de la región Cusco, y que su empleo va más allá del sexo, nivel educativo y nivel socioeconómico.

Una alta proporción de la población encuestada espera que su médico conozca, haga uso y recete plantas medicinales para el tratamiento de dolencias físicas. Creemos que ello se debe a que en Cusco coexisten tres sectores del sistema del cuidado de salud: el sector popular (representado por el ciudadano común); el sector folklórico (representado por curanderos o chamanes), y el sector profesional (representado por profesionales de la salud) (7); este contexto cultural determinaría la elevada frecuencia de uso de plantas medicinales solas o en combinación con medicamentos. Los estudios sugieren que la cooperación entre la medicina tradicional, en este caso empleo de plantas medicinales, y la medicina occidental es posible y beneficiosa para la población; por el contrario, la falta de cooperación puede generar mal uso de la medicina tradicional por el mismo paciente e incluso por el personal de salud ${ }^{(17,18)}$.

El uso de plantas medicinales con más frecuencia hallado en este estudio (digestivos, urinarios y respiratorios en orden de frecuencia) ha sido ampliamente descritos en la literatura (14-16,19); un trabajo realizado en nueve mercados de la ciudad de Cusco encontró 152 especies distintas de plantas medicinales, $40 \%$ de estas eran empleadas para problemas inflamatorios hepáticos y renales; el $30 \%$ para problemas digestivos, y el $20 \%$ para problemas respiratorios ${ }^{(20)}$.

Respecto al empleo de plantas medicinales por profesionales del sector formal, aun cuando las personas pueden confundir a médicos con otro personal de salud, el $36,8 \%$ de participantes refiere que sus médicos le han recetado plantas medicinales. A pesar que los participantes tienen acceso a los servicios de salud, existe gran predisposición y aceptación del uso de plantas medicinales, más aun si la prescripción está dada por médicos del sistema formal de salud. Existe casi un $70 \%$ de participantes que consumen plantas medicinales además del medicamento, este porcentaje es similar a lo encontrado en otros países ${ }^{(15,16)}$, en el presente estudio no se evalúa si dicho uso combinado fue prescrito por el médico tratante.

Dentro de las limitaciones del presente estudio, se debe señalar que los resultados no pueden extrapolarse a la población del distrito de Cusco, puesto que solo se incluyeron participantes que tienen acceso a los hospitales estudiados. Se puede considerar que los resultados 
de frecuencia de uso hubieran sido mayores de realizar el estudio fuera de un ambiente hospitalario (domicilio o comunidad). Las debilidades del cuestionario fueron que no se exploró si el inicio del empleo de plantas medicinales empezó en la infancia a través de los padres, ello permitiría ahondar en el carácter cultural de este fenómeno; tampoco se identificó las plantas medicinales usadas por los participantes para los problemas más comunes de salud. Sin embargo, se puede considerar que este estudio constituye un primer esfuerzo por determinar la frecuencia de uso de plantas medicinales por usuarios de establecimientos del tercer nivel de atención en Perú, dado que la mayoría de estudios nacionales relacionados a plantas medicinales se enfocan en las propiedades de las plantas medicinales.

En conclusión, nuestros resultados brindan una importante evidencia del empleo extendido de las plantas medicinales en usuarios del sistema de salud, en especial entre quienes han recibido la sugerencia de un médico y entre los que desean que el médico les prescriba plantas medicinales. Nuestros resultados sugieren que los usuarios esperan que el sistema de salud incorpore las plantas medicinales en su oferta de salud.

Contribuciones de autoría: GOZ, GHC y FR participaron en la concepción y diseño del artículo, redacción y revisión crítica. Además GHC y FR realizaron el análisis e interpretación de datos. GOZ realizó la obtención de financiamiento. ACh, MAB y LCC realizaron la recolección de resultados. FR brindó también asesoría estadística y asesoría técnica o administrativa. Todos los autores participaron en la revisión crítica del artículo y aprobaron su versión a publicar.

Fuentes de financiamiento: autofinanciado.

Conflictos de interés: los autores declaran no tener conflictos de interés en la publicación de este artículo.

\section{REFERENCIAS BIBLIOGRÁFICAS}

1. Organización Mundial de la Salud. Estrategia de la OMS sobre medicina tradicional 2002-2005. Ginebra: OMS; 2002.

2. Arias B. Diversidad de usos, prácticas de recolección y diferencias según género y edad en el uso de plantas medicinales en Córdoba, Argentina. Bol Latinoam Caribe Plant Med Aromat. 2009;8(5):389-401.

3. Oliveira AK, Oliveira NA, Resende UM, Martins PF. Ethnobotany and traditional medicine of the inhabitants of the Pantanal Negro sub-region and the raizeiros of Miranda and Aquidauna, Mato Grosso do Sul, Brazil. Braz J Biol. 2011;71(1 suppl 1:283--9.

4. Vigano J, Vigano J, Araujo C. Utilização de plantas medicinais pela população da região urbana de Três Barras do Paraná. Acta Sci Health Sci. 2007;29(1):51-8.

5. Llorach C, Chen C, González E, Hernández A, Rodríguez Y. Uso de plantas medicinales en adultos que acuden a una unidad de atención primaria de Panamá. Archivos Med Fam Gen. 2007;4(1):15-8.

6. Primera Cumbre Mundial de Armonización de Medicina Tradicional, Alternativa y Complementaria, Lima, Perú, 2007. Acta Med Per. 2008;25(2):123--4.

7. Mathez-Stiefel SL, Vandebroek I, Rist S. Can Andean medicine coexist with biomedical healthcare? A comparison of two rural communities in Peru and Bolivia. J Ethnobiol Ethnomed. 2012;24;8:26.
8. Sanz-Biset J, Cañigueral S. Plant use in the medicinal practices known as "strict diets" in Chazuta valley (Peruvian Amazon). J Ethnopharmacol. 2011;137(1):271-88.

9. Bussmann RW, Sharon D. Traditional medicinal plant use in Northern Peru: tracking two thousand years of healing culture. J Ethnobiol Ethnomed. 2006;2:47.

10. Bussmann RW, Sharon D, Vandebroek I, Jones A, Revene Z. Health for sale: the medicinal plant markets in Trujillo and Chiclayo, Northern Peru. J Ethnobiol Ethnomed. 2007;3:37.

11. Li Pereyra E. Estado del Arte del Sector de Plantas Medicinales en Perú. Informe Final. Lima: Andean Producs; 2006.

12. Bussmann RW, Sharon D. Shadows of the colonial past--diverging plant use in Northern Peru and Southern Ecuador. J Ethnobiol Ethnomed. 2009;5:4.

13. Ceuterick M, Vandebroek I, Pieroni A. Resilience of Andean urban ethnobotanies: a comparison of medicinal plant use among Bolivian and Peruvian migrants in the United Kingdom and in their countries of origin.J Ethnopharmacol. 2011;136(1):27-54.

14. Picking D, Younger N, Mitchell S, Delgoda R. The prevalence of herbal medicine home use and concomitant use with pharmaceutical medicines in Jamaica. J Ethnopharmacol. 2011; 137(1):305-11.

15. Knotek K, Verner V, Chaloupkova P, Kokoska L. Prevalence and use of herbal products in the Czech Republic: over- the-counter survey among adult pharmacies clients. Complement Ther Med. 2012;20(4):199-206.

16. Gardiner P, Graham R, Legedza AT, Ahn AC, Eisenberg DM, Phillips RS. Factors associated with herbal therapy use by adults in the United States. Altern Ther Health Med. 2007;13(2):22-9.

17. Calvet-Mir L, Reyes-García V, Tanner S. Is there a divide between local medicinal knowledge and Western medicine? a case study among native Amazonians in Bolivia. J Ethnobiol Ethnomed. 2008;4:18.

18. Giovannini P, Reyes-García V, Waldstein A, Heinrich M. Do pharmaceuticals displace local knowledge and use of medicinal plants? Estimates from a crosssectional study in a rural indigenous community, Mexico. Soc Sci Med. 2011;72(6):928-36.

19. Allabi AC, Busia K, Ekanmian V, Bakiono F. The use of medicinal plants in selfcare in the Agonlin region of Benin. J Ethnopharmacol. 2011;133(1):234-43.

20. Huamantupa I, Cuba M, Urrunaga R, Paz E, Ananya N, Callalli M, et al. Riqueza, uso y origen de plantas medicinales expendidas en los mercados de la ciudad del Cusco. Rev Peru Biol. 2011;18(3):283-91.

Correspondencia: Jorge Hernández Córdova

Dirección: Urb. El Hogar A9 San Sebastián, Cusco, Perú.

Teléfono: (511) 973266728

Correo electrónico:gusther007@hotmail.com 